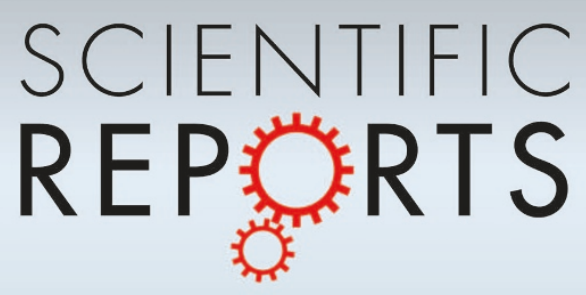

OPEN

SUBJECT AREAS:

NANOSCIENCE AND

TECHNOLOGY

CARBON NANOTUBES AND

FULLERENES

Received

12 June 2014

Accepted

22 September 2014

Published

17 October 2014

Correspondence and requests for materials should be addressed to U.S.C. (uschoi@kist.re.

\section{Line-patterning of polyaniline coated MWCNT on stepped substrates using DC electric field}

\author{
Young Gun Ko', Tae Gu Do², Hyun Chul Oh², Hyun Jeong Lee ${ }^{2}$, Hung-gu Han², Choong Hyun Kim ${ }^{3}$ \\ \& Ung Su Choi ${ }^{2}$
}

\begin{abstract}
'Environmental Radioactivity Assessment Team, Korea Atomic Energy Research Institute, 989-1 11 Daedeok-daero, Yuseong-gu, Daejeon 305-353, Korea, ${ }^{2}$ Center for Urban Energy Systems, Korea Institute of Science and Technology, Hwarangno 14-gil 5, Seongbuk-gu, Seoul 136-791, Korea, ${ }^{3}$ Center for Bionics, Korea Institute of Science and Technology, Hwarangno 14-gil 5, Seongbuk-gu, Seoul 136-791, Korea.
\end{abstract}

Printing electronic components on a chip edge and a stepped substrate with functional inks are an attractive approach for achieving flexible and inexpensive circuits for applications such as flexible displays and large-area chemo/bio/radioactivity sensors. However, it is still challenging because a sufficient cover of the $100 \mu \mathrm{m}$ high step at the chip edge with a high-resolution pattern is the hardest part of the layer assembling by inkjet printing. Herein, we present a simple and effective strategy to generate electrically conductive line-patterns on stepped substrates by applying the DC electric field. On the surface of flat polyimide substrate, the fine line-pattern (less than $850 \mathrm{~nm}$ in line width) is achieved with a polyaniline coated MWCNT dispersed ink. Furthermore, $9.9 \mu \mathrm{m}$ of line width is successfully patterned on the high stepped poly(dimethylsiloxane) substrate, higher than $100 \mu \mathrm{m}$, by printing only 1 time.

$\mathrm{n}$ recent years, a great deal of effort has been expended in the study of the development of inkjet printing technology because of its various applications such as organic thin film transistor, radio-frequency identification devices (RFIDs), light-emitting diodes (LEDs), photovoltaic cells, displays, chemo/bio/radioactivity sensors, etc ${ }^{1}$. This technology has also attracted significant attention as an enabling technology for the rapid, low-cost, scalability to large-area, material-saving, and energy-conserving manufacture of flexible electronics ${ }^{2}$. One of key issues toward "flexible electronics" is to develop the high resolution line-patterning techniques using conducting materials ${ }^{3}$. Therefore, numerous efforts from both scientific and industrial communities have been made to achieve high resolution inkjet-printed features by shrinking the nozzle size ${ }^{4}$ or control of interfacial tension between ink and substrate or nozzle material ${ }^{5}$. However, the high resolution, less than $1 \mu \mathrm{m}$ in line width, has not been achieved with conducting ink such as silver, copper, carbon nanotube (CNT), conducting polymers and indium tin oxide (ITO) by the common inkjet printing technique ${ }^{1,6-8}$ although various materials are patterned one-dimensionally by techniques of several process steps $s^{9,10}$. Recent advance in inkjet printing techniques such as electrostatic autofocussing ${ }^{11}$, electrohydrodynamic jet ${ }^{12}$, electrified liquid jet ${ }^{13}$, and dielectrophoretic nanoprinting ${ }^{14}$ allows the fabrication of high resolution line-pattern which is less than $1 \mu \mathrm{m}$ of line width. Despite the rapid developments in inkjet printing of a conducting ink, printing technology for the step-coverage lateral interconnection over a chip on flexible circuit board still remains a challenging task because the linepatterning is not printed at the side wall of the step. If the standard wire bond process can be replaced by the inkjet technology, we can expect advantages of downsizing possibilities for the chip with better cost performance and shorter interconnects, realization of very complex modules between chips and board connections at small geometric sizes, and easy change of designs for assembling different chip sizes and interconnects by just alterations of the printed program.

Our approach for overcoming the challenging task of printing technology for the step-coverage lateral interconnection over a chip is to apply a DC electric field along the desired direction around an ink-jetted droplet on the substrate. The key of our manufacturing concept is the introduction of electrorheological (ER) phenomena for conductive line-patterning. The ER fluid is a colloidal suspension composed of an insulating liquid medium embodying either a polarizable particulate material or a polarizable liquid material ${ }^{15}$. Application of an electric field to ER particles induces the formation of chains of particles in the colloidal suspension along the field direction ${ }^{16}$. Galliker et al. applied the electric field perpendicularly to the substrate for the fabrication of the high 
resolution line-pattern ${ }^{11}$. The perpendicularlly applied electric field enables the delivering subfemtoliter ink volumes and the high control of ink spreading on the substrate. This method allows the linepattern width down to $1 \mu \mathrm{m}$. However the printing of the lateral line-pattern on the stepped substrate had not been tried by the method. Here, based on the ER phenomena, we demonstrate the fabrication of polyaniline coated multi-walled carbon nanotube (MWCNT@PANI) line-patterning with line width of less than $1 \mu \mathrm{m}$, and with various step heights on the flexible substrates using a commercial needle nozzle. Under the electric field, MWCNT@ PANI particles in the dropped ink on the substrate align along the direction of the electric field with fast evaporation of ink solvent, and finally form the conductive line-pattern.

To the best of our knowledge, this is the first report on the conductive line-patterning on the stepped substrate by the inkjet printing technique. Although our developed technique is an immature technogy, this technique has the potential to be broadly utilized to produce complex patterns and multiple chips on flexible substrates for a wide variety of applications, including in optical, electronic, optoelectronic, and biomedical devices.

\section{Results}

Alignment of MWCNT@PANI particles under DC electric fields. Carbon nanotube (CNT) composites have drawn much attention for electronic devices due to their high electric conductivity ${ }^{17-20}$. Although the drop-on-demand technology, such as inkjet printing, is powerful approach to fabricate fine-electronic devices with high resolution, the technology demands nanometer-sized particles as a dispensing material for small dispensing nozzle diameter. Therefore, a micrometer-length CNT cannot be printed on the circuit-substrate with sub-micrometer scale. In this study, to circumvent this problem, a DC electric field is applied parallel to the substrate for dispensing CNT on the substrate with a commercial needle nozzle. For homogeneous dispersion of CNTs in the ink, CNT was coated with polyaniline (PANI) which is one of the most prominent members of the family of intrinsically conducting polymers ${ }^{21}$. The polyaniline coated multi-walled carbon nanotube (MWCNT@PANI) was prepared by the following procedure (Supplementary Fig. S1). MWCNTs are hydrolyzed for the adsorption of monomer (aniline). And then, oxidative PANI polymerization occurs on the surface of MWCNTs. Without the use of surfactants or stabilizers, our method allows the uniform thickness of PANI layer on MWCNT. The thickness of the PANI layer of the prepared MWCNT@PANI was ca. $3 \sim 7$ nm (Supplementary Fig. S2).

The behavior of MWCNT@PANI in the fluid against DC electric fields was observed using an optical microscope with 5 vol\% of MWCNT@PANI/silicone oil suspension (Fig. 1a). The experimental cell was assembled by mounting two parallel electrodes with a $1.5 \mathrm{~mm}$ gap on a glass slide, in which a drop of well-mixed MWCNT@PANI suspension was dispersed. The presence of fibrils is obvious as the electric field increases, although they are not always linear and even have double loops in some cases. This suspension is called as an electrorheological (ER) fluid. Generally, two different mechanisms have been proposed to explain the ER phenomena ${ }^{22}$. The electrostatic polarization mechanism, proposed originally by Winslow, attributes the origin of the ER effect to the field-induced polarization of the disperse phase particles relative to the continuous phase (Supplementary Fig. S3).

Based on this result, we have tried to confirm the behavior of MWCNT@PANI particles in the droplet of MWCNT@PANI/chlorobenzene under the DC electric field. A conductive line-pattern on the glass substrate has been fabricated as described schematically in Fig. 1b. A suspension is prepared by dispersing MWCNT@PANI in chlorobenzene $(0.5 \mathrm{wt} \%)$. A droplet of the suspension is dropped between the two-electrodes using a commercial needle nozzle. Experiments were carried out with two cases: case 1) evaporation of the droplet without the application of DC electric field, and case 2) evaporation of the droplet after the application of DC electric field.

In the case 1 of Fig. $1 \mathrm{~b}$ (electric field: $0 \mathrm{~V} \mathrm{~mm}^{-1}$ ), a ring composed of MWCNT@PANIs was observed in the field-emission gun scanning electron microscope (FEG-SEM) image (Fig. 1c, left image). Most of solute (MWCNT@PANI) is deposited at the periphery of the dispensed ink due to the strong evaporation flux at the contact line. When the sessile droplet is pinned during the evaporation process, the evaporation flux gradient induces a strong radial outward convective flow, which forces most of the particles to be deposited near the contact line (formation mechanism of a ring-like deposit in Fig. 1b, top inset). This phenomenon is known as the coffee-ring effect ${ }^{23,24}$. In the case 2 experiment of Fig. $1 \mathrm{~b}$, the pattern composed of MWCNT@PANIs depended on the strength of the DC electric field. The diagram of electric field lines around the droplet of MWCNT@PANI suspension is depicted with vector arrows in Fig. 1b, bottom inset ${ }^{25}$. At $250 \mathrm{~V} \mathrm{~mm}^{-1}$, the pattern followed the curvature of the electric field lines (Fig. 1c, middle image). The similar pattern was reported that the domain orientation in the diblock copolymer thin film can be obtained by the application of DC electric field ${ }^{26}$. However, we firstly report that the large pattern is also able to mimic the DC electric field lines on milimeter-length scale. At the higher electric field $\left(500 \mathrm{~V} \mathrm{~mm}^{-1}\right)$, the thick line pattern composed of MWCNT@PANIs was faricated (Fig. 1c, right image). The strongest electric field line forms at the shortest distance between electrodes. Thus, MWCNT@PANIs locate on the the shortest distance between electrodes and form the straight line. In this work, only chlorobenzene was used as an ink medium. In addition to this solvent, various solvents can be used as the ink medium. However, the dielectric constant and electric conductivity of the solvent should be lower than those of MWCNT@PANI. The negative electrorheological (ER) effect occurs when the dielectric constant and electric conductivity of the host fluid are higher than those of the suspended particle (eg, MWCNT@PANI in this work) ${ }^{27}$. In the negative ER fluid, the suspended particles do not form the strong fibrillation parallel to the electric fields because of two phenomena of negative ER fluids; Quincke rotation (electrorotation) and phase separation (electromigration) ${ }^{28}$. The dielectric constant of the used host fluid (chlorobenzene) and particle (polaniline/MWCNT) are 5.69 and 100 $\sim 600$, respectively ${ }^{29-31}$. The electric conductivity of the used particle is also significantly higher than that of host fluid. The host fluid should be evaporized at the room temperature and the atmospheric pressure to fabricate line-pattern in the DC electric field. Supplementary Figure S4 shows the 3D reconstructed images and the height profiles (red lines in the $3 \mathrm{D}$ reconstructed images) of the patterns composed of the MWCNT@PANIs on the glass substrate at the application of 0,250 , and $500 \mathrm{~V} \mathrm{~mm}^{-1}$. At $500 \mathrm{~V} \mathrm{~mm}^{-1}$, the line cross-sectional profile became a roughly convex shape with a high height while other cross-sectional profiles exhibited a low height.

The resistivity of the patterns decreased with the increase of the applied electric field because the MWCNT@PANIs became the denser nanostructure and connected well with each other with increasing the applied electric field as shown in two magnified FEG-SEM images (Supplementary Fig. S4). This dense nanostructure strongly influenced the resistivity. The electric field forced the electrostatically polarized MWCNT@PANIs to be very close to each other, and made them the dense nanostructure. The resistivity of the line-pattern fabricated at $500 \mathrm{~V} \mathrm{~mm}^{-1}$ is $854 \pm 99 \mu \Omega \mathrm{cm}$. This value is significantly lower than that of PANI nanofiber pattern (ca. $1 \Omega \mathrm{cm}$ ), and locates between those of bulk of PANI/PANI salts (ca. $2000 \sim$ $10^{5} \mu \Omega \mathrm{cm}$ ) and carbon nanotubes $(5 \sim 50 \mu \Omega \mathrm{cm})^{32-34}$ (Fig. 2).

High resolution conductive line-patterning on polyimide films. Between two parallel electrodes, single high-resolution line was not obtained due to overlapped electric field lines between the electrodes (see Fig. 1a,c). To address this problem, we did same experiment with 
a
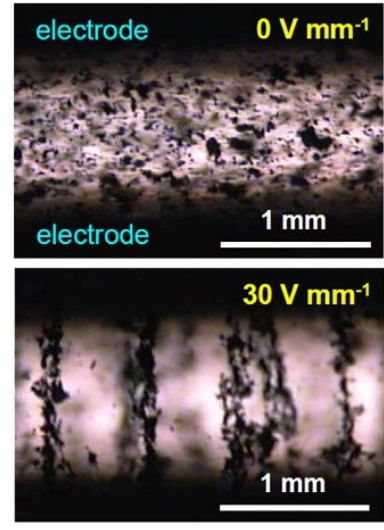

b
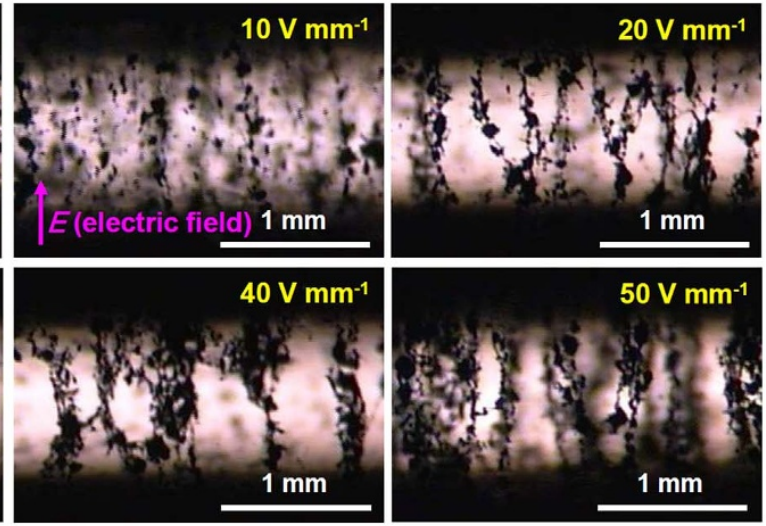
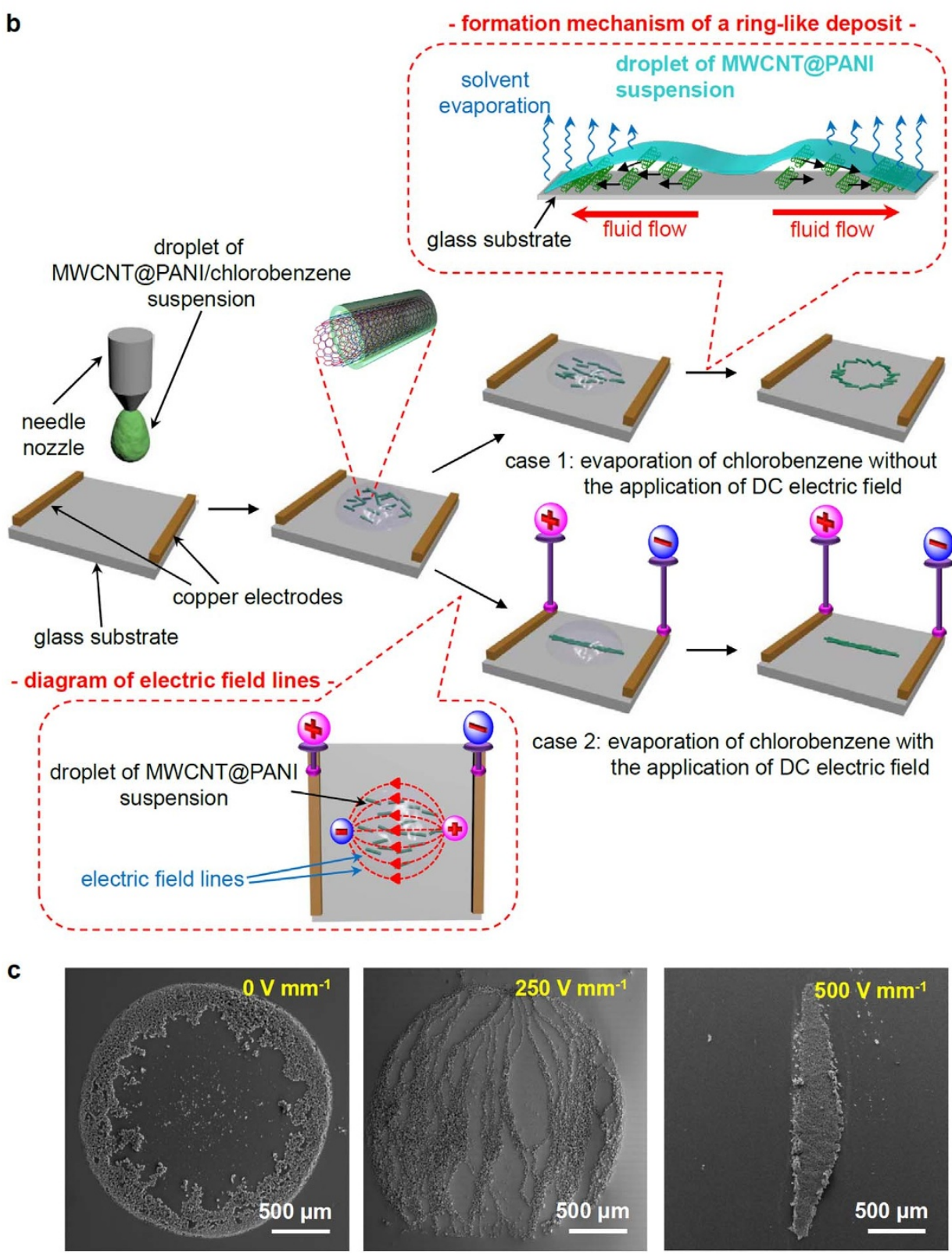

Figure 1 Fabrication of MWCNT@PANI pattern under DC electric field. (a) Optical microscope images of aligned polyaniline-coated multi-walled carbon nanotube (MWCNT@PANI) between two electrodes after application of DC electric fields (gap between electrodes: 1.5 mm, concentration of suspension: 5 vol\% of MWCNT@PANI in silicon oil). (b) Schematic illustration of patterning of MWCNT@PANI on a substrate under DC electric fields. (c) Patterns of MWCNT@PANI on glass substrates under various DC electric fields. 


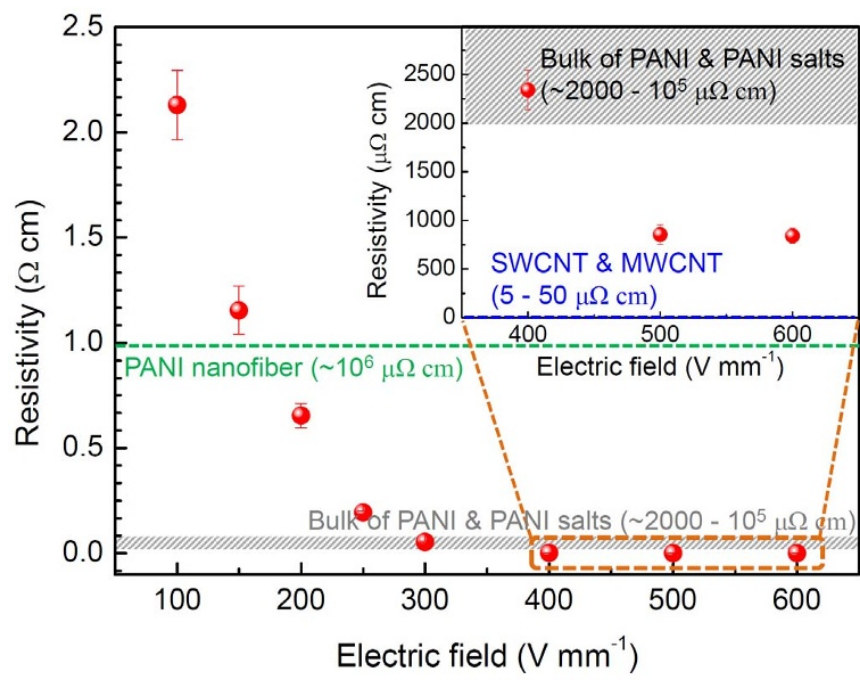

Figure $2 \mid$ Resistivity of fabricated MWCNT@PANI patterns on glass substrates under various DC electric fields. Resistivity significantly decreases with increase of applied DC electric fields. For comparison, resistivity values of PANI nanofiber, bulk of PANI/PANI salts, and carbon nanotubes are represented as a green dashed line $\left(\sim 10^{6} \mu \Omega \mathrm{cm}\right)$, a gray area $\left(\sim 2000-10^{5} \mu \Omega \mathrm{cm}\right)$, and a blue dashed line $(5-50 \mu \Omega \mathrm{cm})$ respectively. Inset is an expanded view of the orange dotted rectangular region. Data are presented as mean \pm standard deviation, $n=5$.

using two needle electrodes instead of two parallel rod-electrodes. In this experiment, single MWCNT@PANI line formed between the electrodes in the silicone oil (Supplementary Movie 1). Inspired by this experimental result, we dropped the MWCNT@PANI/ chlorobenzene solution $\left(1.5 \times 10^{-3}\right.$ wt $\left.\%\right)$ between the sharp-edged copper-electrode patterns on the flexible polyimide film, and then

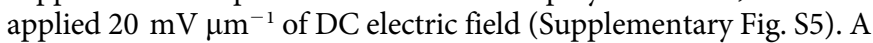
high-resolution conductive line has been fabricated on the flexible polyimide film, and its width and length were $800 \sim 900 \mathrm{~nm}$ and ca. $100 \mu \mathrm{m}$, respectively (Fig. 3). The each MWCNT@PANI tube in the fabricated line aligned well along the direction of the electric field line. This alignment was not observed in high concentration MWCNT@PANI/chlorobenzene (see Supplementary Fig. S4). In the low concentration, tubes stretch along the direction of electric field, and then adhere each other between two electrodes. However, in the high concentration, tubes agglomerate together first due to their polarization and then adhere each other. Due to the dense structure, the fabricated line-pattern showed very low electrical resistivity $(142 \mu \Omega \mathrm{cm})$.

In addition, we fabricated a long conductive line-pattern on the glass substrate with MWCNT@PANI. The conductive line-pattern has been fabricated by repeating steps of 1) dropping of the MWCNT@PANI/chlorobenzene solution, and 2) evaporation of benzene with the application of DC electric field, as described in Supplementary Fig. S6a. To form the long conductive line-pattern, the electrode of the needle type is used, and one electrode is fixed on the glass substrate while another moves in the designed pattern direction. The applied DC electric field for the needle electrodes was $50 \mathrm{~V} \mathrm{~mm}^{-1}$. It is lower than that for the rod electrode type (copper electrodes in Fig. 1b). At the same DC electric field, the electric field line between the needle electrodes is stronger than between the rod electrodes, because only one strong-straight electric-field-line forms between two points while many strong-straight electric-field-line form two between lines. The dimension of the contact between the needle electrode and the glass substrate is zero (point) while the rod electrode is one (line). With the described instrument (Supplementary Fig. S6b), the first initials of the institute (KIST, Korea Institute of Science and Technology) were written on the glass
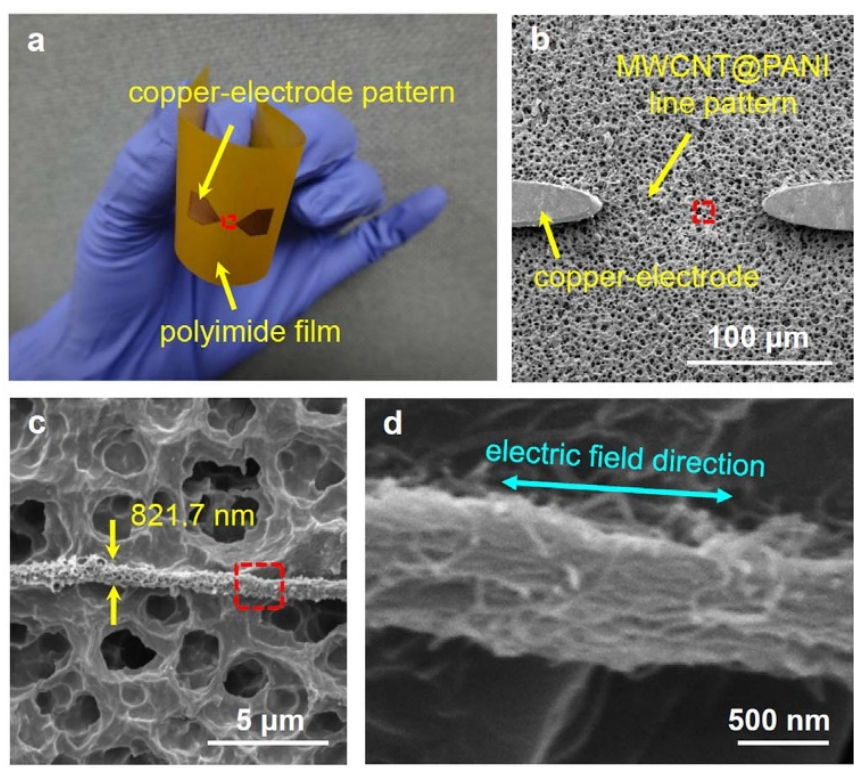

Figure 3 Line patterning of MWCNT@PANI on a polyimide film. (a) Photograph of MWCNT@PANI line pattern on the polyimide (PI) film. Gap between two copper-electrodes is $100 \mu \mathrm{m}$. (b) FEG-SEM image of a red rectangular region in (a). Pores on the PI film formed during etching of the PI film to enhance the copper-electrode adhesion on the film. The pore diameter is $\sim 1 \mu \mathrm{m}$. (c) FEG-SEM image of a red rectangular region in (b). (d) FEG-SEM image of a red rectangular region in (c). Each MWCNT@PANI in the line pattern aligns along the direction of the applied electric field.

substrate as shown in Supplementary Fig. S6c. The dense structure of MWCNT@PANIs in the formed "KIST" is observed (Supplementary Fig. S6d). To see the letters with naked eyes, high concentration of MWCNT@PANI/chlorobenzene (0.5 wt\%) was used to fabricate the initials.

Conductive line-patterning on stepped PDMS films. The drop-ondemand technique offers high technology for the fabrication of modules with multiple chips on a board by printing multilayers on the board with functional inks ${ }^{35}$. However, the technique does not allow the $3 \mathrm{D}$ printing on the stepped surface for the interconnection between chips and board because the printed conductive line disconnects at the sidewall of chip. Our printing technique has been applied to a stepped PDMS substrate (Fig. 4a). The linepattern was fabricated by dropping the MWCNT@PANI/ chlorobenzene solution on the step using a commercial needle nozzle, and appling a DC electric field with two copper needle elecrodes. One was located on the top floor of the stepped film, and the other was on the bottom floor (Fig. 4b). Line widths of 9.9, 50.3 and $70.9 \mu \mathrm{m}$ were fabricated on the stepped PDMS substrates (step height: 105.1, 201.1 and $326.6 \mu \mathrm{m}$ ) at the MWCNT@PANI/ chlorobenzene concentration of $2.5 \times 10^{-3}, 5 \times 10^{-3}$ and $8 \times$ $10^{-3}$ wt $\%$ under DC electric fields of 30,40 and $50 \mathrm{mV} \mu \mathrm{m}^{-1}$, respectively. The data in the graph were obtained by adjusting MWCNT@PANI/chlorobenzene concentration and DC electric field during line-patterning and measuring the line width of the best fine line-pattern. It is noteworthy that the line width shows the repetition of linear increase in range [1] and plateau in range [2] with increase of the step height. In the case of the fabrication of line-patterning on the substrate, applied DC electric field for the patterning is influenced by the electric properties of substrate surface and air. On the other hand, in the case of the stepped substrate, the DC electric field is influenced not only by the substrate surface and the air but the substrate material because the 


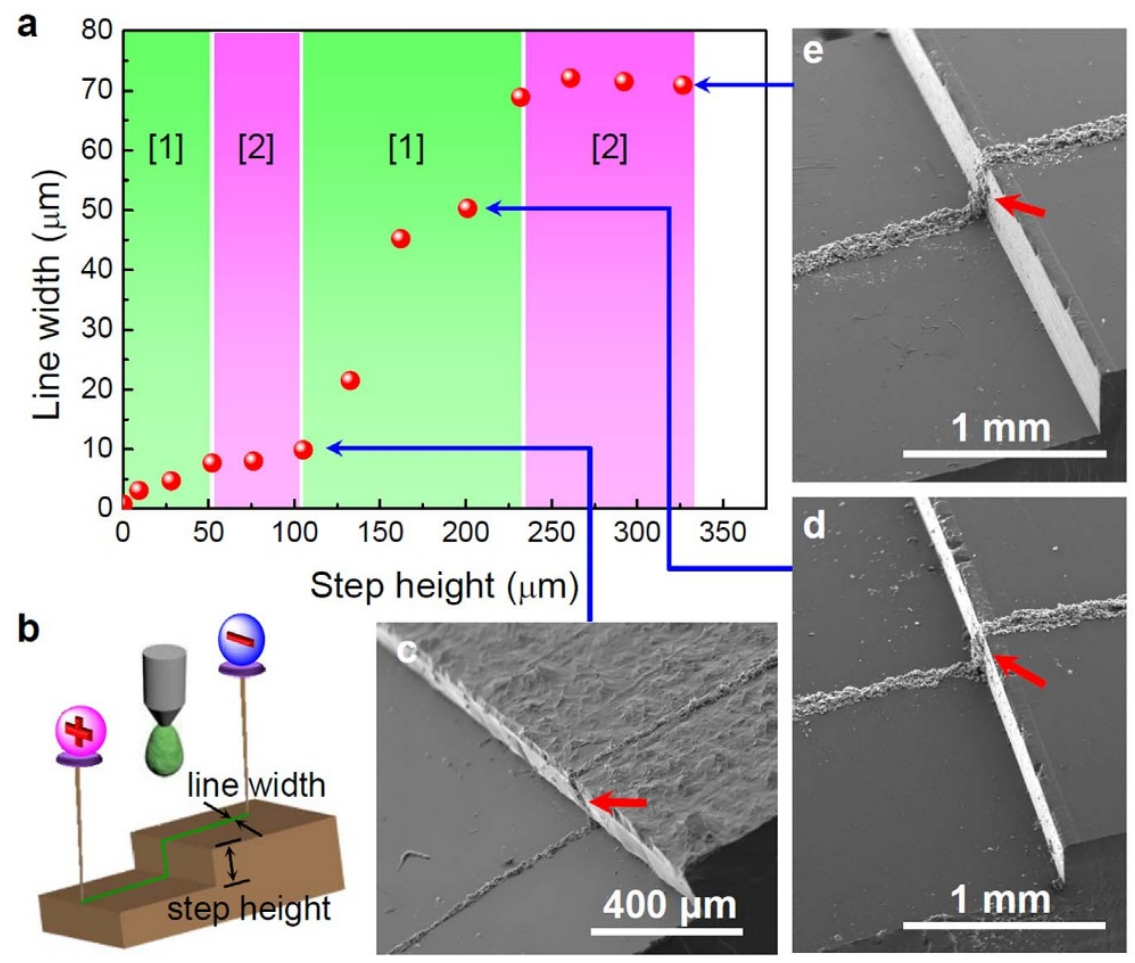

Figure 4 Line patterning of MWCNT@PANI on a stepped PDMS film. (a) Line width of MWCNT@PANI line pattern versus step height of the PDMS film. (b) Schematic illustration of line patterning of MWCNT@PANI on a stepped PDMS substrate under DC electric fields. FEG-SEM images shown in the graph correspond to the fabricated line patterns (c to e). Red arrows indicate a sufficient cover of the high step on the PDMS substrate.

DC electric field should penetrate through the substrate due to the step of the substrate. Thus, a step-height threshold forms due to the resistivity from the substrate, and can be broken by increasing the DC electric field. During increasing the DC electric field, the MWCNT@ $\mathrm{PANI} /$ chlorobenzene concentration is adjusted to avoid the linepattern disconnection and fabricate the fine line-pattern. From this result, to apply our technique to other substrates, the electric properties of the substrates should be considered because the exhibited linear increase (range [1]) and plateau (range [2]) in the graph depend on the electric properties of the substrates.

The fabricated line-patterns exhibit well interconnection at the sidewall of the step with low resistivity (ca. $150 \sim 200 \mu \Omega \mathrm{cm}$ ) to allow the lateral interconnection between chips and board (Fig. 4ce). Hence, the current shortcomings (the high-step-coverage lateral interconnection between chips and a board, and the fine printing of large-sized particle) that plague the application of the drop-ondemand or inkjet technology to industrial fields are eliminated by this innovative approach.

\section{Discussion}

Although the inkjet printing technology is simple, cost-effective, scalable and energy-savable compared with other patterning technologies, it has not been applied for the step-coverage lateral interconnection over a chip or the wiring between semiconductor chips stacked due to the risk of short circuits. Several researches were reported for the step-coverage lateral interconnection over a chip with the inkjet printing technology ${ }^{36,37}$. However, they did not show a printed line with narrow width over a $100 \mu \mathrm{m}$ high step. Herein, we have demonstrated a simple and effective strategy for the first time to generate electrically conductive MWCNT@PANI line-patterns on the stepped substrate by applying the DC electric field. The applied DC electric field kept the line-pattern during evaporation of the ink solvent without its disconnection, and made the fine pattern at the edge of the step of substrate. If the conductivity or the dielectric constant of the material for the patterning is lower than the ink host fluid, Quincke rotation (electrorotation) or phase separation (electromigration) occurs ${ }^{28}$ and prevents the formation of the fine linepattern. Therefore, MWCNT was coated with PANI to increase its dielectric constant although it is a high conductive material. In this study, on the surface of flat polyimide substrate, the line-pattern having the line width of $821.7 \mathrm{~nm}$ and conductivity of $142 \mu \Omega \mathrm{cm}$ was achieved by applying the DC electric field of $20 \mathrm{mV}^{-1} \mathrm{~m}^{-1}$. The each MWCNT@PANI tube in the fabricated line aligned well along the direction of the electric field line. It is noteworthy that a line width of $70.9 \mu \mathrm{m}$ was fabricated on the stepped PDMS substrate (step height: $326.6 \mu \mathrm{m}$ ). A sufficient cover of the $100 \mu \mathrm{m}$ high step at the chip edge is the hardest part of the layer assembling by inkjet printing. Especially, using our method, line width of $9.9 \mu \mathrm{m}$ was patterned on the $105.1 \mu \mathrm{m}$ high stepped PDMS substrate without the cutoff of the MWCNT@PANI line.

In conclusion, the high-resolution line-pattern can be fabricated by the DC electric field assisted inkjet printing technique with the conductive and dielectric material dispersed ink. The technique allows the high step-coverage lateral interconnection over a chip or the wiring between semiconductor chips stacked with the narrow line width. Compared to recent-advanced inkjet printing techniques for the fabrication of the flexible devices, great advantages of our technique are (a) the fabrication of fine pattern with a large particles dispersed ink, (b) the sufficient cover of the high step at the chip edge by printing only 1 time, and (c) the use of commercial printingnozzle (needle nozzle) without adjustment of its size or wettability. It is assumed that with proper optimization of the nanotube concentration, process temperature and applied DC electric field, our suggested method will become a mature and competitive technology for fabricating future fast and low-cost devices, for example, flexible electronic devices and complex chemo/bio/radioactivity sensors.

\section{Methods}

Synthesis of MWCNT@PANI. $1.5 \mathrm{~g}$ of MWCNT (Aldrich) is dissolved in $250 \mathrm{ml}$ of diethyl glycol dimethyl ether (anhydrous, Sigma-Aldrich) and sonicated for $1 \mathrm{~h}$.

$8 \mathrm{~g}$ of 3-sulfolene (Tokyo Chemical Industry) is added into the solution and sonicated 
for $1 \mathrm{~h}$, then reacted at $150^{\circ} \mathrm{C}$ for $24 \mathrm{~h}$ under $\mathrm{N}_{2}$ atmosphere. After the reaction, the reaction mixture is cooled to a room temperature, then reacted with $10 \mathrm{wt} \%$ of $\mathrm{KMnO}_{4}$ (Sigma-Aldrich) aqueous solution in an ice-bath for $24 \mathrm{~h}$. The hydrolized MWCNT is rinsed several times with $0.5 \mathrm{M}$ of $\mathrm{H}_{2} \mathrm{SO}_{4}$ (Sigma-Aldrich) solution, deionized water $(18.2 \mathrm{M} \Omega \cdot \mathrm{cm}$, Milli-Q water system), and ethanol (Sigma-Aldrich) in sequence. Then $0.2 \mathrm{~g}$ of the rinsed-hydrolyzed MWCNT and $150 \mathrm{ml}$ of $1.0 \mathrm{M} \mathrm{HCl}$ solution (Sigma-Aldrich) are transferred into a $500 \mathrm{ml}$ four-neck flask, then sonicated for $1 \mathrm{~h}$ in an ice-bath. $100 \mathrm{ml}$ of $1.0 \mathrm{M} \mathrm{HCl}$ solution containing $0.5 \mathrm{ml}$ of aniline monomer (Sigma-Aldrich) is added into the reaction mixture, then sonicated for $1 \mathrm{~h}$. A $50 \mathrm{ml}$ of $1.0 \mathrm{M} \mathrm{HCl}$ solution containing $1.5 \mathrm{~g}$ of ammonium persulfate (APS, Sigma-Aldrich) is slowly added dropwise into the suspension with constant mechanical stirring in the ice-bath for $15 \mathrm{~min}$. The reaction mixture is stirred for a further $2 \mathrm{~h}$ in the ice-bath, and then the resulting green suspension is filtered and rinsed several times with deionized water and methanol. The powder thus obtained is freezed-dried for 48 h. MWCNT@PANI exhibited obvious absorption corresponding to the chemical structure of green emeraldine hydrochloride in Fourier transform infrared (FT-IR) spectrum (Frontier, PerkinElmer). FT-IR (KBr, $\mathrm{cm}^{-1}$ ): 1565 (quinonoid ring vibration), 1487 (benzenoid ring vibration), 1295 ( $\pi$ electron delocalization), $1239\left(\mathrm{C}-\mathrm{N}^{+}\right.$stretching vibration $)$, and $1121\left(-\mathrm{NH}^{+}=\right.$ stretching vibration).

\section{Observation of structural patterns of MWCNT@PANI particles in silicone oil} under DC electric fields. The experimental cell was assembled by mounting two parallel copper-electrodes with a $1.5 \mathrm{~mm}$ gap on a glass slide, in which a drop of wellmixed MWCNT@PANI/silicone oil (KF-96-50CS, Shin-Etsu Chemical) suspension ( $5 \mathrm{vol} \%$ ) was dispersed. The behavior of the suspension was observed under $0 \sim 50 \mathrm{~V}$ $\mathrm{mm}^{-1}$ using a digital optical microscope (Reichert Metaplan 2, Leica) and a voltmeter (Model 248 High Voltage Supply, Keithley).

Fabrication of patterns on glass substrates. A suspension is prepared by dispersing 0.5 wt\% of MWCNT@PANI in chlorobenzene (Sigma-Aldrich). A droplet of the suspension is dropped between two parallel copper-electrodes (height $\times$ width $\times$ length: $1 \mathrm{~mm} \times 1 \mathrm{~mm} \times 1 \mathrm{~cm}$, distance between electrodes: $5 \mathrm{~mm}$ ) using a commercial needle nozzle (KOVAX-NEEDLE, Korea Vaccine Co., LTD.: 18 G, $19 \mathrm{~mm}$ length, $300 \mu \mathrm{m}$ ID, $450 \mu \mathrm{m}$ OD). Experiments are carried out with two cases: case 1) evaporation of the droplet without the application of DC electric field, and case 2) evaporation of the droplet after the application of DC electric field using a voltmeter. The 3D surface structures and the height profiles of the fabricated patterns can be calculated based on the three FEG-SEM images by finding the height of every point afterwards. Commercial software MeX (Alicona Imaging Co.) was used for the calculation. The electrical resistance of the fabricated patterns was measured with a multimeter (34410A, Agilent) in four-point probe mode. The electrical resistivity $(\rho)$ of the patterns was calculated using the following formula: $\rho=R A / L$, where $R$ is the electrical resistance, $A$ is the cross-sectional area of the pattern and $L$ is the length of the pattern.

Patterning of line electrodes on polyimide substrates. The electrodes-patterned polyimide film was supplied by KM TECH Co. (Korea). The electrodes-patterned polyimide film was prepared by etching a copper-coated polyimide film with a pattern mask. The gap distance between copper-electrodes is $100 \mu \mathrm{m}$, and their width is $30 \mu \mathrm{m}$. The conductive line pattern has been fabricated finely on the flexible polyimide film by following steps; 1) dropping the MWCNT@PANI/chlorobenzene solution $\left(1.5 \times 10^{-3} \mathrm{wt} \%\right)$ using a commercial needle nozzle, and 2) evaporation of chlorobenzene with the application of a DC electric field $\left(20 \mathrm{mV} \mu \mathrm{m}^{-1}\right)$.

Patterning of line electrodes on stepped PDMS substrates. The poly(dimethylsiloxane) (PDMS) substrate was fabricated with elastomer and curing agent. $10 \mathrm{ml}$ of silicone elastomer-184 (Dow Corning) and $1 \mathrm{ml}$ of silicone elastomer curing agent-184 (Dow Corning) were mixed in the $15 \mathrm{ml}$ of Corning tube (Sigma) for about $2 \mathrm{~min}$ at room temperature, and then poured into the silicon wafer ( $\mathrm{P}$ doped ( $\left.\begin{array}{lll}1 & 0 & 0\end{array}\right)$, LG Siltron Inc.) attached petri dish (Aldrich). The uncured PDMS was degassed in a vacuum chamber at ca. $60 \mathrm{mmHg}$ for $1 \mathrm{~h}$. The degassed PDMS was cured in a convection oven at $80^{\circ} \mathrm{C}$ for $4 \mathrm{~h}$. The thick PDMS film was detached from the silicon wafer using a blade. The various step heights were formed by cutting the edge of PDMS films by the blade. The conductive line pattern has been fabricated on the stepped PDMS film by appling DC electric field with two copper needle elecrodes. One was located on the top of the stepped film, and the other was on the bottom.
7. Caironi, M., Gili, E., Sakanoue, T., Cheng, X. \& Sirringhaus, H. High yield, single droplet electrode arrays for nanoscale printed electronics. ACS Nano 4, 1451-1456 (2010).

8. Zhang, Z. et al. Controlled inkjetting of a conductive pattern of silver nanoparticles based on the coffee-ring effect. Adv. Mater. 25, 6714-6718 (2013).

9. Su, B., Wu, Y. \& Jiang, L. The art of aligning one-dimensional (1D) nanostructures. Chem. Soc. Rev. 41, $7832-7856$ (2012).

10. Kan, X., Su, B. \& Jiang, L. Precisely patterning graphene sheets through a liquidbridge induced strategy. Small 10, 2570-2577 (2014).

11. Galliker, P. et al. Direct printing of nanostructures by electrostatic autofocussing of ink nanodroplets. Nat. Commun. 3, 890 (2012).

12. Park, J. et al. High-resolution electrohydrodynamic jet printing. Nat. Mater. 6, 782-789 (2007).

13. Park, J. et al. Nanoscale, electrified liquid jets for high-resolution printing of charge. Nano Lett. 10, 584-591 (2010).

14. Schirmer, N. C. et al. On ejecting colloids against capillarity from sub-micrometer openings: On-demand dielectrophoretic nanoprinting. Adv. Mater. 22, 4701-4705 (2010).

15. Ko, Y. G. \& Choi, U. S. Gelation of natural polymer dispersed suspensions under electric field. Soft Matter 8, 253-259 (2012).

16. Halsey, T. C. Electrorheological fluids. Science 258, 761-766 (1992).

17. Lau, P. H. et al. Fully printed, high performance carbon nanotube thin-film transistors on flexible substrates. Nano Lett. 13, 3864-3869 (2013).

18. Lee, S. Y. et al. Scalable complementary logic gates with chemically doped semiconducting carbon nanotube transistors. ACS Nano 5, 2369-2375 (2011).

19. Kordás, K. et al. Inkjet printing of electrically conductive patterns of carbon nanotubes. Small 2, 1021-1025 (2006)

20. Park, S., Vosguerichian, M. \& Bao, Z. A review of fabrication and applications of carbon nanotube film-based flexible electronics. Nanoscale 5, 1727-1752 (2013).

21. Chiang, J.-C. \& Macdiarmid, A. G. 'Polyaniline': Protonic acid doping of the emeraldine form to the metallic regime. Synth. Met. 13, 193-205 (1986).

22. Parthasarathy, M. \& Klingenberg, D. J. Electrorheology: Mechanisms and models. Mater. Sci. Eng. R. 17, 57-103 (1996).

23. Deegan, R. D. et al. Capillary flow as the cause of ring stains from dried liquid drops. Nature 389, 827-829 (1997).

24. Kawase, T., Sirringhaus, H., Friend, R. H. \& Shimoda, T. Inkjet printed via-hole interconnections and resistors for all-polymer transistor circuits. Adv. Mater. 13, 1601-1605 (2001).

25. Kirkup, L. Computer simulation of electric field lines. Phys. Educ. 20, 142-145 (1985).

26. Morkved, T. L. et al. Local control of microdomain orientation in diblock copolymer thin films with electric fields. Science 273, 931-933 (1996).

27. Ramos-Tejada, M. M., Arroyo, F. J. \& Delgado, A. V. Negative electrorheological behavior in suspensions of inorganic particles. Langmuir 26, 16833-16840 (2010).

28. Ko, Y. G. \& Choi, U. S. Negative electrorheological fluids. J. Rheol. 57, 1655-1667 (2013).

29. Lide, D. R. Handbook of Organic Solvents 77-77 (CRC Press, Boca Raton, 1995).

30. Zhang, Y. et al. Dielectric percolative composites with high dielectric constant and low dielectric loss based on sulfonated poly(aryl ether ketone) and a-MWCNTs coated with polyaniline. J. Mater. Chem. C 1, 4035-4041 (2013).

31. Shi, S.-L., Zhang, L.-Z. \& Li, J.-S. Electrical and dielectric properties of multiwall carbon nanotube/polyaniline composites. J. Polym. Res. 16, 395-399 (2009).

32. Zhang, X. et al. Synthetic process engineered polyaniline nanostructures with tunable morphology and physical properties. Polymer 53, 2109-2120 (2012).

33. Mark, J. E. Polymer Data Handbook 271-275 (Oxford University Press, New York, 1999).

34. Mittal, V. Carbon nanotubes: An introduction. Polymer Nanotube Nanocomposites: Synthesis, Properties, and Applications, Mittal, V. (ed.) 1-13 (Scrivener Publishing, Salem, 2010).

35. Sirringhaus, H. et al. High-resolution inkjet printing of all-polymer transistor circuits. Science 290, 2123-2126 (2000).

36. Mäntysalo, M. et al. System integration of smart package using printed electronics. IEEE Electro. Components and Tech. Conf. (ECTC) 62nd, 997-1002 (2012).

37. Mengel, M. \& Nikitin, I. Inkjet printed dielectrics for electronic packaging of chip embedding modules. Microelectro. Eng. 87, 593-596 (2010).

\section{Acknowledgments}

This work was supported by the Green City Technology Flagship Program funded by the Korea Institute of Science and Technology (KIST-2014-2E25084).

\section{Author contributions}

Y.K. designed experiments and interpreted the experimental findings. T.D. and H.O. developed the fabrication protocols and carried out the fabrication of the patterns. H.L. performed the observation of MWCNT@PANI particles in silicone oil under DC electric fields. H.H. and C.K. contributed to measure the electric properties of the fabricated patterns. Y.K. and U.C. wrote the manuscript, which was revised by all authors. U.C. was responsible for project coordination. 


\section{Additional information}

Supplementary information accompanies this paper at http://www.nature.com/ scientificreports

Competing financial interests: The authors declare no competing financial interests.

How to cite this article: Ko, Y.G. et al. Line-patterning of polyaniline coated MWCNT on stepped substrates using DC electric field. Sci. Rep. 4, 6656; DOI:10.1038/srep06656 (2014). (i) This work is licensed under a Creative Commons Attribution-NonCommercialNoDerivs 4.0 International License. The images or other third party material in this article are included in the article's Creative Commons license, unless indicated otherwise in the credit line; if the material is not included under the Creative Commons license, users will need to obtain permission from the license holder in order to reproduce the material. To view a copy of this license, visit http:// creativecommons.org/licenses/by-nc-nd/4.0/ 
SUBJECT AREAS: NANOSCIENCE AND TECHNOLOGY CARBON NANOTUBES AND FULLERENES

SCIENTIFIC REPORTS:

$4: 6656$

DOI: $10.1038 /$ srep06656

(2014)

Published: 17 October 2014

Updated: 18 March 2015
ERRATUM: Line-patterning of polyaniline coated MWCNT on stepped substrates using DC electric field

Young Gun Ko, Tae Gu Do, Hyun Chul Oh, Hyun Jeong Lee, Hung-gu Han, Choong Hyun Kim \& Ung Su Choi

The original HTML version of this Article contained a typographical error in the spelling of the author Tae Gu Do which was incorrectly given as Tae Gu. This has now been corrected in the HTML version of the Article. 